\title{
Vacancy-Induced Niobate Perovskite-Tungsten Bronze Composite for Synergetic Tuning of Ferroelectricity and Band Gaps
}

\author{
Yang Bai,* Andrey A. Kistanov, Wei Cao, and Jari Juuti
}

Cite This: J. Phys. Chem. C 2021, 125, 8890-8898

Read Online

ABSTRACT: Niobate perovskites like the (K,Na) $\mathrm{NbO}_{3}(\mathrm{KNN})$ family are among the most important lead-free ferroelectrics. $\mathrm{Ba}$ and $\mathrm{Ni}$ have been co-doped into KNN to induce $\mathrm{Ni}^{2+}$-oxygen vacancy defect dipoles to significantly reduce the band gap while maintaining the ferroelectricity. This opens doors to novel optoferroelectric applications such as multisensors and photocatalysts. However, obtaining a single phase of the above co-doped KNN is difficult due to the sensitive stoichiometry on phase formation and arduous nickel diffusion into the KNN unit cells during synthesis. This paper reports an alternative approach to simultaneously tune the band gap and ferroelectricity. A-site vacancies are intentionally introduced into the mixtures of starting reactants. The

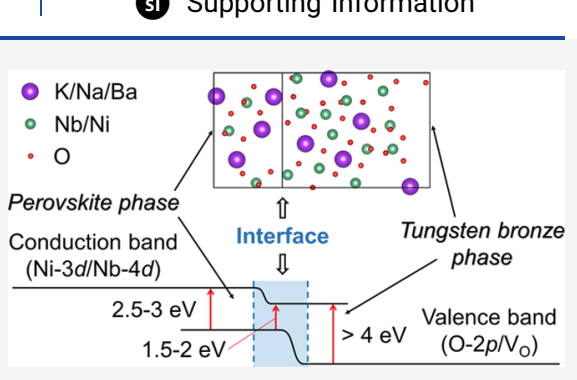
homogeneously distributed vacancies trigger self-assembly of a niobate perovskite and niobate tungsten bronze phase and thus form a composite. The interface between the two phases, which mimics a heterojunction, rather than any individual phase, is proven to be responsible for the resultant narrow band gap and strong ferroelectricity. Hypotheses are proposed based on the results of ferroelectric, photoconductivity, and density functional theory-based studies to explain the mechanism. This paper offers an additional option to engineer polarizations and band structures in complex photoferroelectric oxides, especially alkaline niobates.

\section{INTRODUCTION}

Ferroelectrics, among polar dielectrics with switchable spontaneous polarizations (namely, ferroelectricity) in unit cells and domains, are widely used in electro-mechanothermal coupling components like memories, detectors, and transducers. ${ }^{1,2}$ Although most ferroelectrics are considered as insulators or semiconductors with wide optical band gaps beyond the majority of photon energy of visible light (e.g., $>2.7 \mathrm{eV}$ ), their band gaps can be engineered as small as $1.1 \mathrm{eV}$ (same as that of $\mathrm{Si}$ ). ${ }^{3}$ Ferroelectrics may also exhibit a photovoltaic effect, and given proper conditions, they are able to deliver above band gap, ultra-high photovoltages and a photovoltaic energy conversion efficiency that breaks the physical (Shockley-Queisser) limit predicted for conventional semiconductor solar cells. ${ }^{4-6}$

Most high-performance ferroelectrics have $\mathrm{ABO}_{3}$ perovskite structures. The $\mathrm{d}^{0}$ transition metals on the $\mathrm{B}$-sites possess necessary electronic structures for the second-order JahnTeller distortion, driving the B-site cations to displace from the center positions in the O-octahedra and thus giving rise to ferroelectricity. ${ }^{7}$ However, the large electronegativity difference between the two ends of the $\mathrm{B}-\mathrm{O}$ bonds leads to a high charge transfer energy from $\mathrm{O} 2 \mathrm{p}$ states to the B-site metal $\mathrm{d}$ states, resulting in a large band gap. ${ }^{7}$ Pioneer studies suggested that by properly introducing non- $\mathrm{d}^{0}$ metal states into the B-sites as well as pairing them with $\mathrm{O}$ vacancies $\left(\mathrm{V}_{\mathrm{O}}\right)$, the large band gap in $\mathrm{ABO}_{3}$ ferroelectric perovskites could be effectively reduced. For instance, $\mathrm{KNbO}_{3}$ has been doped with $10-40 \mathrm{~mol} \%$ $\mathrm{Ba}\left(\mathrm{Ni}_{0.5} \mathrm{Nb}_{0.5}\right) \mathrm{O}_{3-\Delta}$ (BNNO), which could naturally provide both $\mathrm{Ni}^{2+}$ and $\mathrm{V}_{\mathrm{O}}{ }^{3}$ The $\mathrm{Ni}^{2+}-\mathrm{V}_{\mathrm{O}}$ defect dipoles significantly eased the charge transfer (band-band transition) and impressively reduced the band gap of $\mathrm{KNbO}_{3}$ by up to 2.7 $\mathrm{eV}$ (from 3.8 to $1.1 \mathrm{eV}$ ). ${ }^{3}$ Although polarizations were suppressed to a negligible level at room temperature in the $\mathrm{KNbO}_{3}-\mathrm{BNNO}$, a follow-up work has proven that by precisely controlling and limiting the $\mathrm{BNNO}$ dopant to $2 \mathrm{~mol} \%$ and by introducing $\mathrm{Na}^{+}$on the A-site to help increase unit cell distortion, the doped $\left(\mathrm{K}_{0.5} \mathrm{Na}_{0.5}\right) \mathrm{NbO}_{3}$ reduced the band gap by up to $2.4 \mathrm{eV}$ (from 4 to $1.6 \mathrm{eV}$ ) and simultaneously retained the remanent polarization at $11 \mu \mathrm{C} \mathrm{cm}^{-2}$ (55\% of that of the undoped $\left.\left(\mathrm{K}_{0.5} \mathrm{Na}_{0.5}\right) \mathrm{NbO}_{3}\right){ }^{8}$

Nevertheless, in the research community, using Ni doping to reduce band gaps of niobate perovskites is under debate. It is questionable how effective is the $\mathrm{Ni}^{2+}-\mathrm{V}_{\mathrm{O}}$ doping strategy, especially when being transferred to other fabrication routes or compositions. For instance, standalone $\mathrm{NiO}$ was found in both ceramic samples made via solid-state reaction and nanoparticles synthesized by the sol-gel method for the $\mathrm{KNbO}_{3}$ BNNO and $\left(\mathrm{K}_{0.5} \mathrm{Na}_{0.5}\right) \mathrm{NbO}_{3}$-BNNO compositions. ${ }^{3,8-10}$ Meanwhile, in ceramic samples of $\mathrm{BaTiO}_{3}$ doped with $\mathrm{BNNO}$, a $\mathrm{Ni}-\mathrm{V}_{\mathrm{O}}$-rich composition at grain boundaries was observed to be favored while only a small amount of $\mathrm{Ni}$ was

Received: March 1, 2021

Revised: March 31, 2021

Published: April 15, 2021 
diffused into the grains where it was expected to be. ${ }^{11}$ These pieces of evidence indicate that it is difficult to form a single perovskite phase with the desired $\mathrm{Ni}$ concentration on the $\mathrm{B}$ site of the unit cells, probably due to different radii of $\mathrm{Ni}^{2+}$ and $\mathrm{Nb}^{5+}$ ions. ${ }^{9}$ Furthermore, when being adopted to compositions like $(\mathrm{Na}, \mathrm{Bi}) \mathrm{TiO}_{3}-\mathrm{BaTiO}_{3}$ and $(\mathrm{K}, \mathrm{Na}, \mathrm{Li})(\mathrm{Nb}, \mathrm{Ta}) \mathrm{O}_{3}-\mathrm{CaZrO}_{3}$, the $\mathrm{Ni}^{2+}-\mathrm{V}_{\mathrm{O}}$ doping caused multiple absorption peaks, which was thought to be attributed to the in-gap states created by $\mathrm{Ni}$ between the conduction band minimum formed by $\mathrm{Nb} 4 \mathrm{~d}$ states and valence band maximum formed by $\mathrm{O} 2 \mathrm{p}$ states. ${ }^{12,13}$ In other words, the $\mathrm{Ni}^{2+}-\mathrm{V}_{\mathrm{O}}$ in fact did not ease the bandband transition and thus did not reduce the actual band gap, in contrast to that observed in $\mathrm{KNbO}_{3}$-BNNO and $\left(\mathrm{K}_{0.5} \mathrm{Na}_{0.5}\right)$ $\mathrm{NbO}_{3}$-BNNO. ${ }^{3,8}$ The discrepancy implies that the $\mathrm{Ni}^{2+}-\mathrm{V}_{\mathrm{O}}$ doping strategy is considerably composition-dependent, thus imposing obstacles to developing a universal strategy for band gap engineering for ferroelectric perovskites.

In this paper, an alternative route of simultaneously tuning ferroelectricity and band gaps is presented. During the research of the $\mathrm{Ni}^{2+}-\mathrm{V}_{\mathrm{O}}$ doping in $\left(\mathrm{K}_{0.5} \mathrm{Na}_{0.5}\right) \mathrm{NbO}_{3}$, the authors accidentally found a perovskite-tungsten bronze composite, which also showed surprisingly an effective reduction of the band gap and increase of spontaneous and remanent polarizations compared to the single perovskite phase counterpart. After implementing a comprehensive and systematic investigation, the phenomenon observed in the new composite is confirmed. The composite shares the same fabrication method with the previously reported single-phase perovskite, but instead of having stoichiometric starting reactants, different concentrations of A-site deficits are intentionally introduced into the nominal chemical formula of the starting reactants. Within a certain window of the A-site deficit concentration, a composite containing a perovskite phase and a tungsten bronze phase having the same types of elements but different molecular ratios between the elements and microstructures is grown from the homogeneously mixed starting reactants. Neither the perovskite nor the tungsten bronze phase is able to individually reach the optimum ferroelectricity and narrowest band gap (measured in this research), but the composite as a whole can achieve this. With studies of the ferroelectric hysteresis loop and photoconductivity on both the phases, the interface between them is concluded to be responsible for the rise of polarizations and ease of charge transfer. Band bending and/or misalignment at the phase interface region are suggested to be the reasons supported by density functional theory (DFT) calculations. The approach of the perovskitetungsten bronze composite in this paper may provide hints for potential further work on other compositions such as $\mathrm{KNbO}_{3}$, $\mathrm{BaTiO}_{3},\left(\mathrm{Bi}_{0.5} \mathrm{Na}_{0.5}\right) \mathrm{TiO}_{3}$, and their families to optimize their photoferroelectric properties.

\section{EXPERIMENTAL SECTION}

2.1. Sample Fabrication. Ceramic samples were fabricated via the solid-state route. The concentrations of the starting reactants were calculated based on the nominal chemical formula $\left(\mathrm{K}_{i}, \mathrm{Na}_{j}, \mathrm{Ba}_{k}\right)\left(\mathrm{Nb}_{l}, \mathrm{Ni}_{m}\right) \mathrm{O}_{3-\delta}$ (KNBNNO). In this formula, the B-site (value of $l+m$ ) was normalized to 1 while the A-site (value of $x=i+j+k$ ) varied from 1.05 (5 mol $\%$ extra compared to the stoichiometric one) to $0.8(20 \mathrm{~mol} \%$ in deficit). This consideration was made based on the fact that each $\mathrm{Ba}^{2+}$ may replace one or two $\mathrm{K}^{+}$(or $\mathrm{Na}^{+}$) in reality, which could not be controlled during high-temperature reaction. ${ }^{14}$ The value of $3-\delta$ was calculated by neutralizing the net chemical valence, i.e., $3-\delta=(i+j+2 k+5 l+2 m) / 2$. Figure 1 shows the distribution maps of $x$ and $3-\delta$ values in this

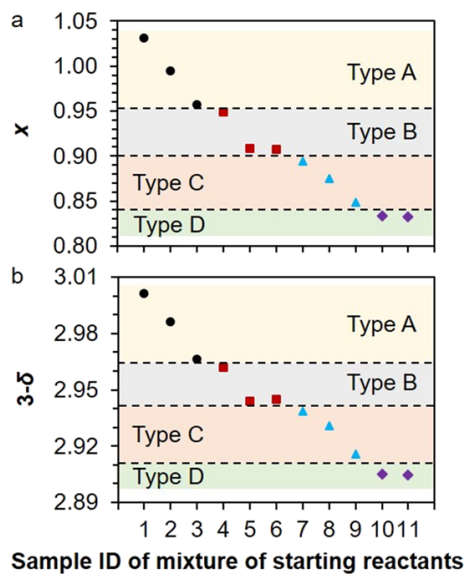

Figure 1. Values of (a) $x(=i+j+k)$ and (b) $3-\delta$ for the mixtures of starting reactants determined by XRF.

research. Figures $\mathrm{S} 1$ and $\mathrm{S} 2$ in the Supporting Information details the distributions of $i, j, k, l$, and $m$ values as well as their ratios. Tables S1 and S2 in the Supporting Information list the actual weighed concentrations and amounts of the starting reactants. Table 1 summarizes the nominal chemical formulae of the four types of starting reactant mixtures marked in Figure 1. A detailed description of the fabrication procedures is given in the Supporting Information (Section 1 - Detailed experimental procedures). In brief, the weighed starting reactants were mixed, calcined at $825{ }^{\circ} \mathrm{C}$ for $4 \mathrm{~h}$ in air, shaped into disc green bodies, and sintered at $1150{ }^{\circ} \mathrm{C}$ for $2 \mathrm{~h}$ in a buried and sealed environment. The synthesis conditions were kept identical to all samples.

It should be noted that the values in Figure 1 and Figure S1 were determined with XRF (X-ray fluorescence, ASX S4 Pioneer, Bruker, USA) for the 11 mixtures of starting reactants. For each mixture sample ( $20 \mathrm{~g}$ for each mixture), five random batches of the mixed reactant powders ( $1 \mathrm{~g}$ for each batch) were subjected to XRF tests. The values shown in Figure 1 and Figure $\mathrm{S} 1$ were the averages with deviations of less than $5 \%$. This indicates that the mixing process (see Section 1 of the Supporting Information) was effective and the starting reactants were evenly present in each mixture. Although the ratios between $i, j, k, l$, and $m$ varied across the 11 mixtures as can be seen in Figure S2 (Supporting Information), the types of samples (Type A, Type B, Type C, and Type D) were mainly classified by $x$ and $3-\delta$ values as shown in Figure 1 . The ratios between $i, j, k, l$, and $m$ had only a negligible influence on the microstructure and properties of the finally sintered ceramics. Such results will be shown and discussed in the following parts of this paper.

In these complex compounds, the practical weighing procedure (see Section 1 of the Supporting Information) required special attention. The reactants were first weighed targeting to the desired concentrations and mixed followed by validation under XRF. Due to the daily change of humidity in air, the relatively more hygroscopic $\mathrm{K}_{2} \mathrm{CO}_{3}$ and $\mathrm{Na}_{2} \mathrm{CO}_{3}$ (compared to other reactants) could cause an increased level of deviation from the desired concentrations during weighing. If this happened and was detected by XRF, a minor adjustment (i.e., an increase or decrease of certain concentration in the 
Table 1. Nominal Chemical Formulae of the Mixtures of Starting Reactants

sample type
A
B
C
D

nominal chemical formula

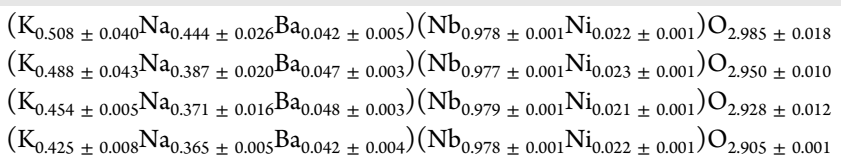

opposite direction to the detected deviation) was introduced and the weighing process was repeated immediately on the same day until the desired concentrations were reached in XRF tests as shown in Figure 1 and Figures S1 and S2.

2.2. Characterization. XRD (X-ray diffraction, D8 Discover, Bruker, USA) was used to identify phase structures. A UV-vis-NIR spectrophotometer (Cary 500 Scan, Varian, USA) was used to characterize the absorption behavior. FESEM (field emission scanning electron microscope, ULTRA plus, Zeiss, Germany) and EPMA (electron probe microanalyzer, JXA-8530FPlus, JEOL, Japan) were used to analyze the microstructures and elements. Ferroelectric hysteresis loops $(1 \mathrm{~Hz})$ and piezoelectric coefficients were measured using a ferroelectric evaluation system (Precision LCII, Radiant Technologies Inc., USA) and a Berlincourt piezoelectric meter (YE2730A, APC International Ltd., USA), respectively. Large-signal conductivity was measured with the same ferroelectric evaluation system. Small-signal conductivity was measured using a four-terminal method with a SourceMeter (model 2450, Keithley, USA). Light sources were provided by three lasers (OBIS LX/LS series, Coherent, USA) with wavelengths of 405, 552, and $660 \mathrm{~nm}$. Detailed characterization methods are described in the Supporting Information (Section 1 - Detailed experimental procedures). It should be noted that in both the conductivity measurements, no matter which phase was illuminated, the conductivity was measured for the entire surface area covered by electrodes.

\section{RESULTS}

3.1. Materials and Structures. All sintered ceramic samples had densities of $4.5-4.6 \mathrm{~g} \mathrm{~cm}^{-3}$ (equivalent to $>98 \%$ relative density). Figure 2 shows the appearances and

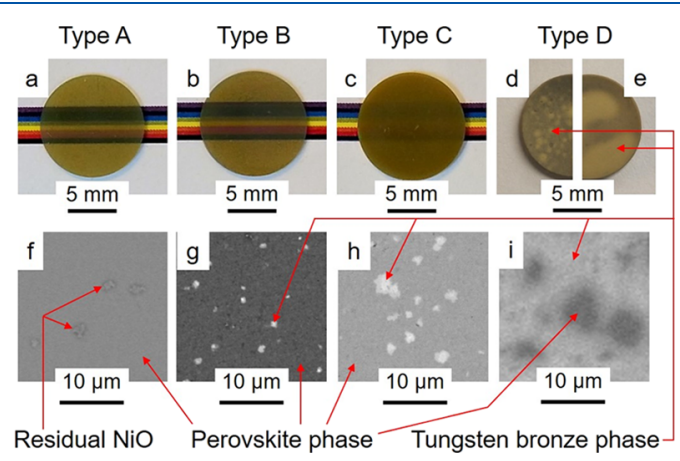

Figure 2. $(a-e)$ Pictures and (f-i) EPMA micrographs (backscattered electron mode) of the (a,f) Type A, (b,g) Type B, $(\mathrm{c}, \mathrm{h})$ Type C, and (d,e,i) Type D ceramics.

microstructures (backscattered electron image obtained with EPMA) of the ceramic samples (finely polished, see Section 1 in the Supporting Information) made from the four types of powder mixtures (as shown in Figure 1 and Figures $S 1$ and S2). In Figure $2 a-c$, the rainbow-like background helps to indicate the transparency where the samples had the same thickness of about $400 \mu \mathrm{m}$ (see Section 1 in the Supporting Information). It can be seen in Figure $2 \mathrm{a}$ that the Type A ceramics were rather transparent with an antique bronze color. Figure $2 \mathrm{f}$ shows that a single perovskite phase (identified later with XRD) was formed in the Type A ceramics with roughly 1 $\mu$ m-sized residual $\mathrm{NiO}$ particles that can be clearly seen on the surface. The residual $\mathrm{NiO}$ did not diffuse into the unit cells as expected, reflecting observations in the literature. . $^{8,10}$

Comparing Figure 2a,b, the transparency difference between Type A and Type B samples was hard to distinguish. However, in Figure 2g, a secondary phase, which was later identified as a tungsten bronze phase, was formed in the perovskite phase matrix. A significant decrease of transparency from Type B to Type $C$ samples can be noticed by comparing Figure 2 b,c. Correspondingly, the size of the tungsten bronze phase increased from $<1 \mu \mathrm{m}$ in Figure $2 \mathrm{~g}$ to $1-3 \mu \mathrm{m}$ in Figure $2 \mathrm{~h}$. The Type D samples shown in Figure 2d,e formed an extended amount of tungsten bronze phase, which could even be easily seen by eyes. This resulted in the majority part shown in Figure $2 \mathrm{i}$ to be the tungsten bronze phase surrounding the perovskite phase, which is opposite to the situation in Figure $2 \mathrm{~h}$ where the tungsten bronze phase was surrounded by the perovskite phase. Although the samples shown in Figure 2d,e had the same values of $x$ and $3-\delta$, the difference between their $\mathrm{Ba}$ concentrations (see Figures $\mathrm{S} 1-\mathrm{S} 2$ in the Supporting Information) caused the sample in Figure $2 \mathrm{~d}$ to form a distributed, smaller tungsten bronze phase while the one in Figure $2 \mathrm{e}$ formed a larger tungsten bronze phase. This difference will be discussed in Section 4 of this paper.

As mentioned above, the perovskite and tungsten bronze phases were identified with XRD patterns shown in Figure 3. Table S3 in the Supporting Information gives full lists of peak positions, $d$-spaces, and peak intensities extracted from the raw data collected from the XRD scans (Figure 3) for the four
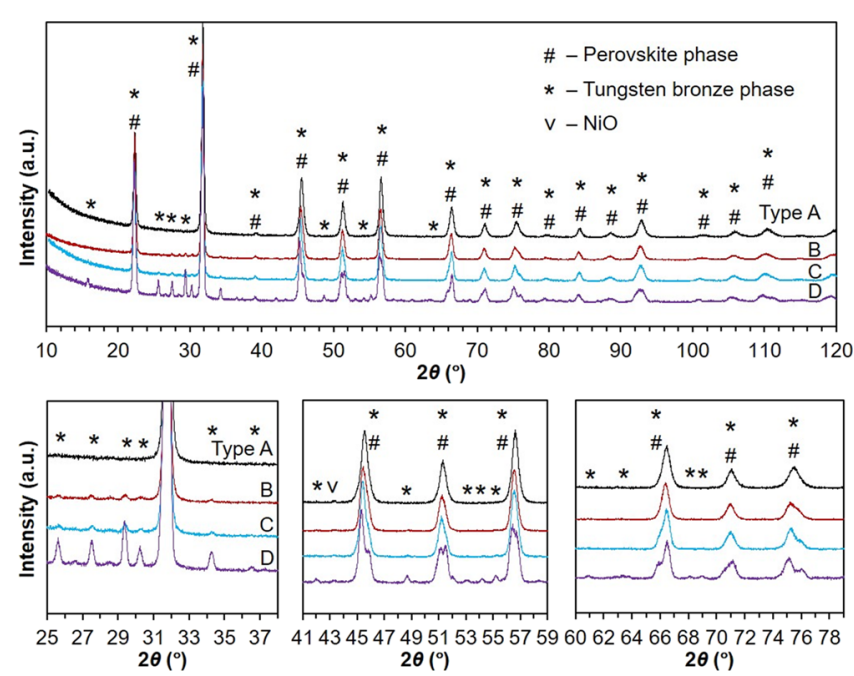

Figure 3. XRD patterns of the Type A, Type B, Type C, and Type D ceramic samples. 
Table 2. Chemical Formulae and Lattice Parameters of the Perovskite Phase and Tungsten Bronze Phase

\begin{tabular}{|c|c|c|c|}
\hline phase & chemical formula & space group & $a, b$, and $c(\AA) ; \alpha, \beta$, and $\gamma\left(^{\circ}\right)$ \\
\hline perovskite & 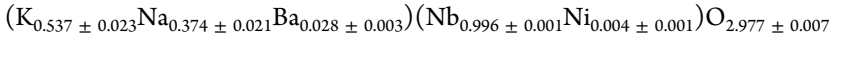 & $\begin{array}{l}\text { Amm } 2 \\
\text { orthorhombic }\end{array}$ & $\begin{array}{l}a=3.9746 \pm 0.0231, b=5.6531 \pm 0.0150, c= \\
5.6652 \pm 0.0176 ; \alpha=\beta=\gamma=90^{\circ}\end{array}$ \\
\hline $\begin{array}{l}\text { tungsten } \\
\text { bronze }\end{array}$ & $\left(\mathrm{K}_{0.251} \pm 0.021 \mathrm{Na}_{0.097} \pm 0.013 \mathrm{Ba}_{0.190} \pm 0.014\right)\left(\mathrm{Nb}_{0.970} \pm 0.003 \mathrm{Ni}_{0.030} \pm 0.003\right) \mathrm{O}_{2.818} \pm 0.011$ & $\begin{array}{l}P 4 b m \\
\text { tetragonal }\end{array}$ & $\begin{array}{l}a=b=12.5351 \pm 0.0037, c=3.9669 \pm 0.0052 \\
\alpha=\beta=\gamma=90^{\circ}\end{array}$ \\
\hline
\end{tabular}

types of ceramic samples. The perovskite phase, tungsten bronze phase, and residual $\mathrm{NiO}$ observed in Figure $2 \mathrm{f}$ to Figure 2i can be clearly identified in Figure 3. The results of Rietveld refinement carried out with a Type D sample, which had the largest concentration of the tungsten bronze phase, hence the most distinguishable calculated peaks, are shown in Figure S3 in the Supporting Information. The indexed peaks after the refinement are also given in Table S3 (Supporting Information). Consistent with the observations in Figure $2 \mathrm{f}$ to Figure $2 \mathrm{i}$, residual $\mathrm{NiO}$ was only detectable in the Type $\mathrm{A}$ samples as shown in Figure 3. This indicates a better diffusion of Ni into the unit cells in the Type B, Type C, and Type D samples (proven and discussed later). It should be noted that, in order to exclude the influence caused by possible surface texture, the ceramic disc samples were crushed into ceramic powders and were re-measured by XRD. The differences in the calculated phase concentrations were found to be negligible.

Together with XRD identification, 10 random spots $(1 \mu \mathrm{m}$ in diameter) on each of the phases in each sample shown in Figure 2 were analyzed by EPMA, and thus, the concentration of each element was assessed. Note that each type of ceramic had three batches (except two batches for Type D, see Figure 1) with each batch having three samples tested, corresponding to the 11 batches shown in Figure 1. This means that 33 samples in total were studied in this research. The chemical formulae obtained by EPMA and the lattice parameters obtained by refinement of the perovskite and tungsten bronze phases are listed in Table 2, and the phase ratio in each type of ceramic is shown in Table 3 . Note that the concentrations of

Table 3. Concentrations of the Perovskite and Tungsten Bronze Phases in Each of the Four Types of Ceramics

$\begin{array}{ccc}\text { sample type } & \text { perovskite phase }(\mathrm{mol} \%) & \text { tungsten bronze phase }(\mathrm{mol} \%) \\ \text { A } & 100 & 0 \\ \text { B } & 92.5 \pm 0.5 & 7.5 \pm 0.5 \\ \text { C } & 82.7 \pm 7.7 & 17.3 \pm 7.7 \\ \text { D } & 57.4 \pm 16.9 & 42.6 \pm 16.9\end{array}$

the tungsten bronze phase were first calculated by refinement but with a nominal chemical formula of (K,Na,$\mathrm{Ba})_{2.9}(\mathrm{Nb}, \mathrm{Ni})_{5.4} \mathrm{O}_{15.2}$. In Tables $2-3$ and Figure $\mathrm{S} 4$, the chemical formulae of both the phases were normalized to $\mathrm{Ni}+$ $\mathrm{Nb}$ (B-site) $=1$ for easier comparison. Therefore, the indicative concentrations listed in Table 3 and elsewhere throughout this paper were obtained from the values calculated from the refinements and then multiplying a factor of 5.4. NiO was ignored in Table 3 because the analysis was inaccurate $(<0.5 \mathrm{~mol} \%)$. The indexes of each individual phase are provided in Table S4 in the Supporting Information. The phase structures are also supplied as CIF files in the Supporting Information 2 and 3. Detailed EPMA results are provided in Figure S4 in the Supporting Information.

As the size of the tungsten bronze phase in Type B samples was smaller than the EPMA spot size (as presented above), the elemental analyses on Type B samples exhibited an increased level of deviations in Figure S4. However, such deviations were proven to be analysis errors caused by the large EPMA spot size through a series of tests at the phase boundary area on a Type C sample, as shown in Figure S5 in the Supporting Information.

3.2. Optical and Electrical Properties. Figure 4 shows the transmittance and absorption coefficients of the Type A,

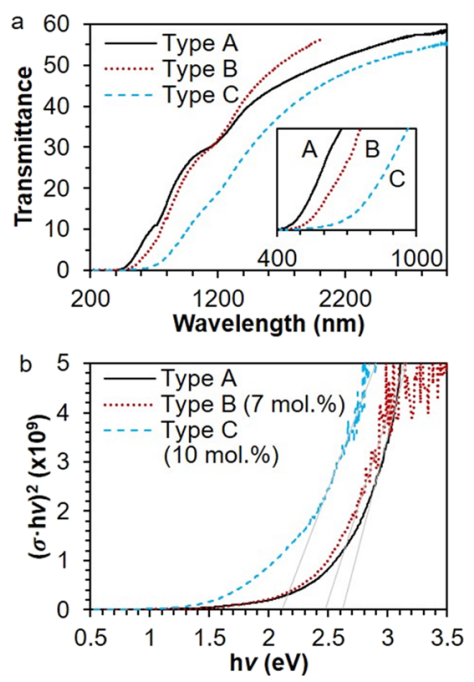

Figure 4. Dependence of (a) transmittance and (b) $(\sigma h \nu)^{2}$ on (a) wavelength and $(\mathrm{b})$ photon energy $(h \nu)$ of incident light for the Type A, Type B, and Type C samples, where $\sigma$ is the absorption coefficient. The concentrations of the tungsten bronze phase ( $\mathrm{mol} \%)$ for the Type B and Type C samples are indicated.

Type B, and Type C ceramic samples. The Type D samples were too opaque for the light sources of the spectrophotometer (light intensities were not high enough) used in this research to penetrate through the samples. As a result, the collected transmittance and absorbance data were like white noises varying around 0 . Therefore, the data obtained from the Type $\mathrm{D}$ samples were not able to make meaningful assessment and thus are not shown here. The Type $C$ ceramics exhibited a clearly different behavior from those of the Type A and Type B samples. As shown in Figure 4a, the transmittances of the Type $A$ and Type B samples started to increase at an incident light wavelength of about $400 \mathrm{~nm}$, compared to $600 \mathrm{~nm}$ for the Type C. Similarly, in Figure $4 b$, the band gaps of the Type A and Type B samples defined by the Tauc fitting were 2.4-2.6 $\mathrm{eV}$ while that of the Type $\mathrm{C}$ was about $2.1 \mathrm{eV}$. Figure $\mathrm{S} 6$ in the Supporting Information shows the grains of the ceramic samples imaged under FESEM. As can be seen in Figure S6, the grain sizes of the perovskite phases in Type $A$ and those of the Type B and Type C samples were similar. Therefore, the changes of transmittance and absorption observed in Figure 4 were likely to be due to the expansion of the tungsten bronze phase. Figure S7 in the Supporting Information plots the square roots of the absorption coefficients, which, together 
with Figure $4 \mathrm{~b}$, indicate that all the samples tended to have direct absorptions.

According to empirical experience, using absorption behavior to define the band gaps of polycrystalline and inhomogeneous materials containing defects or minor dopants is unreliable. For this reason, in this paper, photoconductivity is used to help judge the change of band gaps. Figure 5 plots

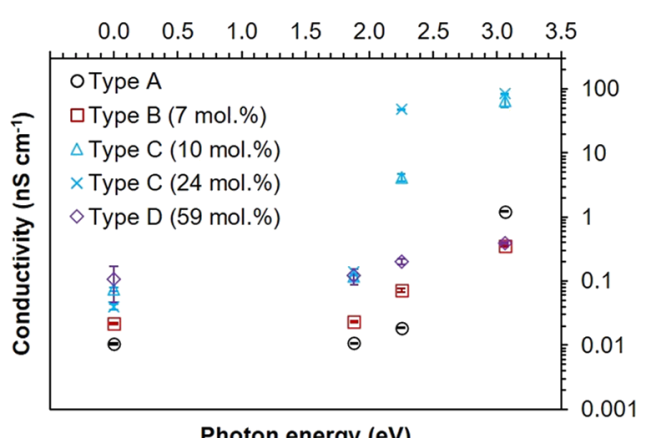

Figure 5. Dependence of conductivity on the photon energy of the incident laser beam for the four types of ceramics. The data points at $0 \mathrm{eV}$ were collected in darkness. The concentrations of the tungsten bronze phase (mol \%) for the Type B, Type C, and Type D samples are marked.

the conductivity of the four types of ceramics measured in darkness $(0 \mathrm{eV})$ as well as under laser illumination. The incident laser beams had an output power of $20 \mathrm{~mW}$ and wavelengths of 660,552 , and $405 \mathrm{~nm}$, corresponding to photon energies of $1.88,2.25$, and $3.06 \mathrm{eV}$, respectively (see Section 1 in the Supporting Information). It should be noted here that the diameters of the laser beams were 700-900 $\mu \mathrm{m}$ (see Section 1 in the Supporting Information), over 100 times the size of the tungsten bronze phase in the Type B and Type C samples (Figure 2g,h and Figure S6). This ensured that both the perovskite and tungsten bronze phases, as well as their interfaces, were illuminated at any random point on the sample surface. However, the laser beams were not necessarily large enough to cover both the phases in Type D samples. The Type D sample shown in Figure 5 possessed a 59 mol \% tungsten bronze phase occupying larger areas than those of the laser beams. This sample is shown in Figure 2e, of which the results shown in Figure 5 were collected when the illuminations were only on the tungsten bronze phase (i.e., the perovskite phase was not illuminated).

In Figure 5, significant increases of conductivity can be seen for the Type A, Type B, and Type C samples under illumination compared to those in darkness $(0 \mathrm{eV})$. In comparison, the conductivity change of the Type D sample (or the tungsten bronze phase to be accurate) was much less noticeable. This implies that the individual tungsten bronze phase was not likely to be able to reduce the band gap. Among the Type B and Type C samples, it seems that the larger concentration of the tungsten bronze phase could induce larger conductivity change as shown in Figure 5. In order to distinguish whether the cumulative effect of both phases or any individual phase was the main reason for the conductivity change, a Type D sample with about $26 \mathrm{~mol} \%$ tungsten bronze phase, which is the sample shown in Figure $2 \mathrm{~d}$, was measured. The evenly distributed, large enough tungsten bronze phase areas among the perovskite phase matrix allowed positioning the laser beams on different spots of the perovskite and tungsten bronze phases in the same sample. Figure S8 in the Supporting Information shows the conductivity of both the individual phases measured in darkness and under illumination. Figure S8 confirms the observation in Figure 5 that an individual tungsten bronze phase was not responsible for the significant conductivity changes in the Type B and Type C samples. Meanwhile, although the conductivity change of the perovskite phase was larger than an order of magnitude from in darkness to under illumination (Figure S8), it was still negligible compared to the 3 orders of magnitude change in the Type B and Type C samples (Figure 5). Therefore, it is likely that the cumulative effect of both the phases was responsible for the significant conductivity change rather than any individual phase. In addition, the perovskite phase in Figure $S 8$ behaved differently from the Type A sample with the single perovskite phase (Figure 5). This was because the smallsized tungsten bronze phase was possible to be present under the perovskite surface and thus degraded the conductivity change compared to that caused by a pure perovskite phase.

Figure 6 compares the ferroelectric hysteresis loops and piezoelectric coefficients $\left(d_{33}\right)$ measured in darkness for the

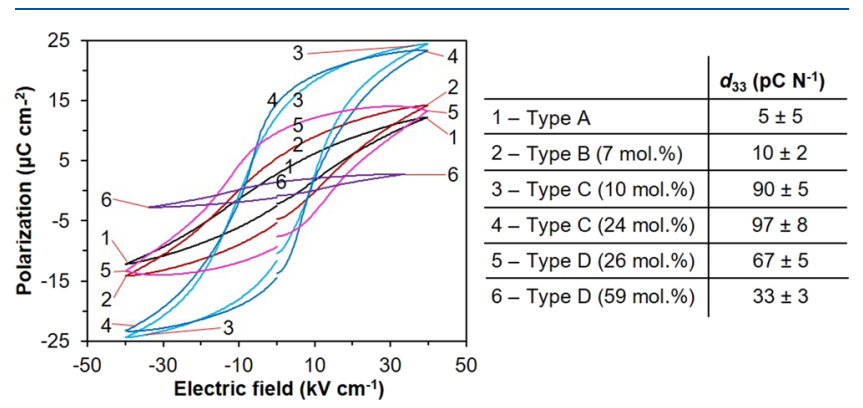

Figure 6. Ferroelectric hysteresis loops and $d_{33}$ values measured in darkness for the four types of ceramics.

four types of ceramics. Similar to that shown in Figure 5, the Type D sample with a $59 \mathrm{~mol} \%$ tungsten bronze phase (Figure $2 \mathrm{e}$ ) in Figure 6 was measured by applying an electric field only on the tungsten bronze phase. The remanent polarization and $d_{33}$ values increased with the increase of the tungsten bronze phase concentration in the sample until approximately $24 \mathrm{~mol}$ $\%$ and then decreased. Figure 6 indicates that neither individual perovskite nor tungsten bronze phase alone was able to maximize the ferroelectric and piezoelectric properties but the cumulative effect of both phases was needed.

To summarize the relationships between the material structures and properties, Figure 7 plots $E_{\mathrm{g}}{ }^{*}, P_{\mathrm{m}}, P_{\mathrm{r}}$, and $d_{33}$ as a function of the tungsten bronze phase concentration, where $P_{\mathrm{m}}$ and $P_{\mathrm{r}}$ are the polarization at the maximum electric field and the remanent polarization (extracted from Figure 6), respectively. $E_{\mathrm{g}}{ }^{*}$ represents the photon energy needed to increase the sample's conductivity by an order of magnitude (extracted from Figure 5). Here, $E_{\mathrm{g}}{ }^{*}$ is used as an alternative method to effectively reflect the band gap. Clearly, $E_{\mathrm{g}}{ }^{*}$ started to decrease with the increase of the tungsten bronze concentration from the $8-10 \mathrm{~mol} \%$ point and reached a minimum of about $2 \mathrm{eV}$ with an approximately $20 \mathrm{~mol} \%$ tungsten bronze phase. After the $20 \mathrm{~mol} \%$ point, $E_{\mathrm{g}}{ }^{*}$ increased rapidly to beyond the visible light range. The trends for $P_{\mathrm{m}}, P_{\mathrm{r}}$, and $d_{33}$ were inverse compared to that of $E_{\mathrm{g}}{ }^{*}$, where the maxima were reached with an approximately $25 \mathrm{~mol} \%$ tungsten bronze phase. A jump and a plunge in the $d_{33}$ curve 


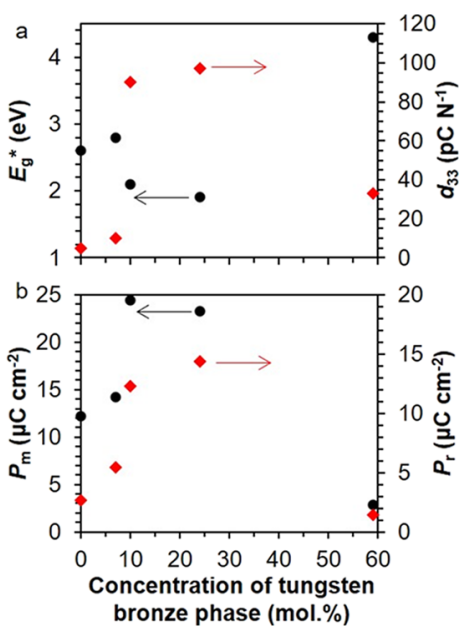

Figure 7. Dependence of (a) $E_{\mathrm{g}}{ }^{*}$ and $d_{33}$ and (b) $P_{\mathrm{m}}$ and $P_{\mathrm{r}}$ on the concentration of the tungsten bronze phase for the $\left(\mathrm{K}_{i}, \mathrm{Na}_{j}, \mathrm{Ba}_{k}\right)$. $\left(\mathrm{Nb}_{b} \mathrm{Ni}_{m}\right) \mathrm{O}_{3-\delta}$ perovskite-tungsten bronze composite samples (data extracted from Figures 5 and 6 ).

can be recognized across the $8-10$ and $24-26 \mathrm{~mol} \%$ regions of the tungsten bronze phase, respectively, implying two critical points between which the optimum $d_{33}$ values could be achieved (namely, with the Type $\mathrm{C}$ samples).

As mentioned in the Experimental Section above and in Section 1 of the Supporting Information, the results shown in Figure 5 were obtained from large-signal measurements where the input electric field was $15 \mathrm{kV} \mathrm{cm}^{-1}$. According to Figure 6, this electric field was slightly higher than the coercive fields of all samples. Such an input electric field for large-signal measurements was chosen in order to minimize the influence of mobile trapped charges/charged defects and leakage current surge during domain wall motion on the conductivity. ${ }^{15-17}$ However, in order to validate the conductivity results, smallsignal measurements (see Section 1 in the Supporting Information) were also carried out. Figure S9 shows the results of a Type $\mathrm{C}$ sample ( $10 \mathrm{~mol} \%$ tungsten bronze phase) measured in darkness and under illuminations. The photon energy needed to reach an order of magnitude increase of the conductivity was also about $2.1 \mathrm{eV}$ for the same sample, consistent with the corresponding results shown in Figure 5. This proves the validity of the large-signal measurements used in this paper.

\section{DISCUSSION}

4.1. Relationship between A-Site Deficits in the Starting Reactants and Phases in the Sintered Ceramics. Formation of the tungsten bronze phase in $\mathrm{KNN}$ perovskite ceramics has been well presented in the literature. ${ }^{18}$ From the evidence shown in Section 3.1 above, it can be concluded that in KNBNNO ceramics, a single perovskite phase would be formed with a maximum $5 \mathrm{~mol} \%$ A-site deficit in the starting reactants (Figure 1). This was independent of the specific ratios between $\mathrm{K}, \mathrm{Na}$, and $\mathrm{Ba}$ concentrations (Figures S1, S2, and S4). However, although the $\mathrm{Ni}$ concentration was about $2 \mathrm{~mol} \%$ of the B-site atoms in the starting reactants (Table 1), only $0.5 \mathrm{~mol} \% \mathrm{Ni}$ (of B-site atoms) could finally diffuse into the perovskite phase to form integrated KNBNNO unit cells (Table 2). With relatively fully occupied A-sites (like Type A ceramics), the extra Ni that could not diffused into the perovskite unit cells was forced to stay as standalone $\mathrm{NiO}$ particles (Figures 2 and 3 ).

However, $\mathrm{Ni}$ showed a much better diffusion into the KNBNNO tungsten bronze phase formed in the Type B, Type $\mathrm{C}$, and Type D ceramics. These samples had corresponding types of starting reactants with increased A-site deficit ( $>5 \mathrm{~mol}$ $\%)$. A reaction and phase formation route can be proposed here:

In these A-site deficient starting reactant mixtures, a KNBNNO perovskite phase was still preferred to be first formed during the solid-state reaction because the tungsten bronze phase expanded only with the A-site deficiency (Figures $1-2$ and Table 3 ). Although there were A-site deficits ( $>5 \mathrm{~mol}$ $\%)$ in the starting reactants, a consistent, stoichiometric KNBNNO perovskite phase could always be formed with $>95$ mol \% of the A-sites occupied, which was the same situation as in the Type A ceramics (Figure S4). This also happened independent of specific ratios between $\mathrm{K}, \mathrm{Na}$, and $\mathrm{Ba}$ concentrations (Figures S1, S2, and S4). These results imply that during the solid-state reaction, the elements diffused actively to prioritize the formation of the stoichiometric KNBNNO perovskite phase, leading to increased levels of Asite deficit in the unreacted regions of the starting reactant mixtures. This forced the rest of the reactants to form a KNBNNO tungsten bronze phase.

Surprisingly, the unit cells of the KNBNNO tungsten bronze phase could easily host $3 \mathrm{~mol} \%$ (compared to only $0.5 \mathrm{~mol} \%$ in the perovskite phase) $\mathrm{Ni}$ on the B-sites (Table 2). This left much less residual $\mathrm{NiO}$ particles in the Type $\mathrm{B}$, Type $\mathrm{C}$, and Type D ceramics. Compared to the perovskite phase, the tungsten bronze phase contained significantly less $\mathrm{K}$ and $\mathrm{Na}$ but more $\mathrm{Ba}$ (Table 2 and Figure $\mathrm{S} 4$ ). As one $\mathrm{Ba}^{2+}$ could replace two $\mathrm{K}^{+}$(or $\mathrm{Na}^{+}$), the high concentration of $\mathrm{Ba}$ in the tungsten bronze phase was likely to leave enough space and tolerance in the unit cell to host more $\mathrm{Ni}$ (radius larger than that of $\mathrm{Nb}$ ). It was also possible that $\mathrm{Ni}$ had a higher diffusion rate in the tungsten bronze phase.

Furthermore, the ratio between $\mathrm{K}$ (or $\mathrm{Na}$ ) and $\mathrm{Ba}$ in the starting reactants was likely to be a crucial factor for the formation of the tungsten bronze phase. In one of the Type D samples (starting reactant mixture ID 10 in Figures S1-S2), the $\mathrm{K} / \mathrm{Ba}$ ratio (i.e., $i / k$ value) was about $9(\mathrm{Na} / \mathrm{Ba}$ or $j / k$ value was about 8 ) while the other Type D sample (mixture ID 11) had an $i / k(j / k)$ value of about 11 (9) (Figure S2). This difference resulted in two different ceramics as shown in Figure $2 \mathrm{~d}$,e where the sample in Figure $2 \mathrm{~d}$ had a smaller amount and distributed tungsten bronze phase compared to that in Figure $2 \mathrm{e}$ with a larger amount and area of the tungsten bronze. In mixtures 10 and 11 , the $\mathrm{K} / \mathrm{Na}$ ratios $(i / k)$ and $\mathrm{K}+\mathrm{Na}$ concentrations $(i+j)$ were similar (Figure S2). This implies that, given the same and high level of A-site deficit ( $>15 \mathrm{~mol}$ $\%$, Figure 1) in the starting reactants, a relatively smaller concentration of $\mathrm{Ba}$ promoted the formation of the large area tungsten bronze phase. A relatively higher $\mathrm{Nb}$ concentration (Figure S2) in the starting reactants seemed to have the same effect. It can then be concluded that the $15 \mathrm{~mol} \%$ A-site deficit in the KNBNNO starting reactants was the boundary beyond which the formation of the tungsten bronze phase became favored.

4.2. Correlation of the Perovskite-Tungsten Bronze Phase Interface and Band Gap/Ferroelectric Properties. In Section 3.2, it has been proven that neither of the individual phases was able to reach the optimum ferroelectric/piezo- 
electric properties and the narrowest achievable band gap. Therefore, a hypothesis is proposed here to explain the cumulative effect of both the perovskite and tungsten bronze phases.

It is known that for the same ferroelectric composition, a larger remanent polarization usually induces a larger piezoelectric coefficient. The change of $d_{33}$ followed the trend of the remanent polarization in Figure 6, indicating a variation of true remanent polarization in the measurements. Meanwhile, ferroelectric hysteresis loops were obtained individually with the single perovskite phase (Type A sample) and mainly tungsten bronze phase (Type D $59 \mathrm{~mol} \%$ ), proving that both the phases contained switchable domains and mobile domain walls. For the tungsten bronze phase, a nonpolar space group, $P 4 / \mathrm{mbm}$, and a polar equivalent, $P 4 \mathrm{bm}$, could both be identified during the refinements. The difference between the two symmetries was negligible for XRD reflections, especially when those intensities of the tungsten bronze phase were rather small. However, the polar $P 4 b m$ was believed to be a preferred ground state of the tungsten bronze phase according to the hysteresis loop measured as well as to some similar examples predicted by calculations. ${ }^{19-21}$

Since the perovskite and tungsten bronze phases had orthorhombic and tetragonal structures, respectively, a pseudo-morphotropic phase boundary could be formed at the interface regions between the phases where the adjacent unit cells gradually evolved from one structure to the other (Figure S5). Formation of morphotropic phase boundaries has been well proven to be an effective approach to optimize ferroelectric and piezoelectric properties in KNN-based compositions. ${ }^{22}$ In this paper, the interface regions between the perovskite and tungsten bronze phases were thought to be responsible for the increased ferroelectric and piezoelectric properties by enhancing the domain wall mobility at the pseudo-morphotropic phase regions. In such regions, all the unit cells were in a transition status and tended to both the orthorhombic perovskite and tetragonal tungsten bronze phases. More possibilities were given to the polarization directions when switching the domains compared to the situations in the individual phases. Particularly, a giant leap of the polarizations and $d_{33}$ was observed at the tungsten bronze phase concentration of approximately $10 \mathrm{~mol} \%$ (Figure 7). This implies that when the tungsten bronze phase was less than $10 \mathrm{~mol} \%$, the phase interface area was not large enough to induce a significant increase of ferroelectric and piezoelectric properties.

Figure 8 proposes a mechanism of the band gap reduction at the phase interface. According to Figures 4, 5, and 7, the band gaps of individual perovskite and tungsten bronze phases were likely to be $2.5-3$ and $>4 \mathrm{eV}$, respectively, as shown in Figure 8 where the valence band may be contributed by $\mathrm{O} 2 \mathrm{p}$ states and/or trapped charges at $\mathrm{V}_{\mathrm{O}}$ that could become mobile under

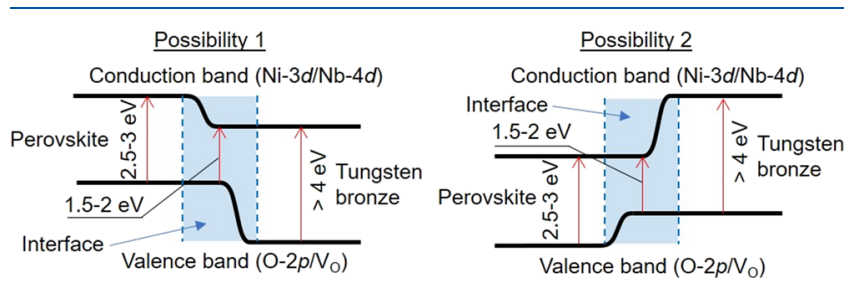

Figure 8. Hypothesis of band bending and misalignment at the perovskite-tungsten bronze phase interface. illumination and/or electric field and the conduction band may be contributed by $\mathrm{Ni} 3 \mathrm{~d}$ and/or $\mathrm{Nb} 4 \mathrm{~d}$ states. ${ }^{3,16,23}$ In the KNBNNO perovskite-tungsten bronze composites fabricated in this research, the conduction and valence bands were possibly bent and/or misaligned at the phase interface in two ways as shown in Figure 8. Such a bent and/or misaligned band structure at the interface allowed the electrons in the valence band of the perovskite phase to be excited to the conduction band of the tungsten bronze phase with photon energy larger than $1.5-2 \mathrm{eV}$ (Possibility 1 in Figure 8 ) or the other way round (Possibility 2 in Figure 8), resulting in largely reduced $E_{\mathrm{g}}{ }^{*}$ values of $1.5-2 \mathrm{eV}$ compared to those of individual phases as shown in Figures 5 and 7. The overlaps of the conduction band minimum and valance band maximum of different phases in Figure 8 are presented to visualize the possible band extensions toward each individual structure in the unit cells of the interface regions that may be in a mixed/ transition status. The real situation of the conduction and valence bands should be expressed by a proper band structure calculation.

To confirm the proposed hypothesis, the band structures of perovskite $\mathrm{KNbO}_{3}$, tungsten bronze $\mathrm{KNb}_{2} \mathrm{O}_{5}$, and their interfaces were studied by DFT calculations. Details of the calculation methods can be found in the Supporting Information (Section 3 - Simulation). Due to the complexity of the unit cells of KNBNNO perovskite and tungsten bronze structures, the models were simplified to be a $\mathrm{KNbO}_{3} / \mathrm{KNb}_{2} \mathrm{O}_{5}$ interface. Figures $\mathrm{S} 10-\mathrm{S} 13$ in the Supporting Information show the atomic structures, band structures, and local densities of states (LDOS) of the perovskite $\mathrm{KNbO}_{3}$, tungsten bronze $\mathrm{KNb}_{2} \mathrm{O}_{5}$, and two kinds of possible interfaces between the phases. According to the results of the calculations, $\mathrm{KNbO}_{3}$ (Figure $\mathrm{S} 10$ ) and $\mathrm{KNb}_{2} \mathrm{O}_{5}$ (Figure $\mathrm{S} 11$ ) had band gaps of 2.46 and $1.75 \mathrm{eV}(0.25 \mathrm{eV}$ with states of $1.5 \mathrm{eV}$ below the Fermi level), respectively. Meanwhile, the LDOS plots in Figures S10 and $\mathrm{S} 11$ indicate that the valence bands of $\mathrm{KNbO}_{3}$ and $\mathrm{KNb}_{2} \mathrm{O}_{5}$ mainly consisted of $\mathrm{O} 2 \mathrm{p}$ states while their conduction bands mainly consisted of $\mathrm{Nb} 4 \mathrm{~d}$ states. Analyses of band structures and LDOS at the two kinds of $\mathrm{KNbO}_{3}$ / $\mathrm{KNb}_{2} \mathrm{O}_{5}$ interfaces, as shown in Figures $\mathrm{S} 12$ and S13, suggest the $\mathrm{O}$ and $\mathrm{Nb}$ originating states were strongly mixed. Consequently, the conduction and valence bands at the interfaces were bent and misaligned, resulting in a decrease or vanishing of the band gap of the $\mathrm{KNbO}_{3} / \mathrm{KNb}_{2} \mathrm{O}_{5}$ composite. The obvious change of the band's alignment at the perovskitetungsten bronze interface revealed in the DFT calculations confirmed the hypothesis shown in Figure 8.

\section{CONCLUSIONS}

By intentionally designing 5-15 mol \% A-site deficit in the starting reactants, composites containing a roughly $80-90 \mathrm{~mol}$ $\%$ perovskite phase (nominal chemical formula $\left.\left(\mathrm{K}_{0.54} \mathrm{Na}_{0.37} \mathrm{Ba}_{0.03}\right)\left(\mathrm{Nb}_{0.996} \mathrm{Ni}_{0.004}\right) \mathrm{O}_{2.979}\right)$ and $10-20 \mathrm{~mol} \%$ tungsten bronze phase (nominal chemical formula $\left.\left(\mathrm{K}_{0.25} \mathrm{Na}_{0.10} \mathrm{Ba}_{0.19}\right)\left(\mathrm{Nb}_{0.97} \mathrm{Ni}_{0.03}\right) \mathrm{O}_{2.82}\right)$ have been fabricated via solid-state reaction. The perovskite and tungsten bronze phases have obtained orthorhombic and tetragonal structures, respectively, likely to form a pseudo-morphotropic phase boundary at the phase interfaces. The phase boundary has boosted the remanent polarization and piezoelectric coefficient by up to 3 and 5 times, respectively, compared to those of the individual phases. The possible band bending and misalignment at the interfaces have also reduced the band gap by 
approximately $1-2 \mathrm{eV}$ compared to the individual phases. This research offers a new, alternative route to simultaneously tune (optimize) ferroelectric/piezoelectric properties and band gaps in inorganic photoferroelectric materials, especially for alkaline niobates.

\section{ASSOCIATED CONTENT}

\section{(s) Supporting Information}

The Supporting Information is available free of charge at https://pubs.acs.org/doi/10.1021/acs.jpcc.1c01845.

Detailed experimental procedures, supplementary data, and simulation (PDF)

CIF file for $\left(\mathrm{K}_{0.54} \mathrm{Na}_{0.37} \mathrm{Ba}_{0.03}\right)\left(\mathrm{Nb}_{0.996} \mathrm{Ni}_{0.004}\right) \mathrm{O}_{2.979}$ (CIF)

CIF file for $\left(\mathrm{K}_{0.25} \mathrm{Na}_{0.10} \mathrm{Ba}_{0.19}\right)\left(\mathrm{Nb}_{0.97} \mathrm{Ni}_{0.03}\right) \mathrm{O}_{2.82}$ (CIF)

\section{AUTHOR INFORMATION}

\section{Corresponding Author}

Yang Bai - Microelectronics Research Unit, Faculty of Information Technology and Electrical Engineering, University of Oulu, FI-90014 Oulu, Finland; 이이.org/ 0000-0002-0861-2410; Email: yang.bai@oulu.fi

\section{Authors}

Andrey A. Kistanov - Nano and Molecular Systems Research Unit, Faculty of Science, University of Oulu, FI-90014 Oulu, Finland; (1) orcid.org/0000-0001-6175-5296

Wei Cao - Nano and Molecular Systems Research Unit, Faculty of Science, University of Oulu, FI-90014 Oulu, Finland; $\odot$ orcid.org/0000-0003-3139-1780

Jari Juuti - Microelectronics Research Unit, Faculty of Information Technology and Electrical Engineering, University of Oulu, FI-90014 Oulu, Finland

Complete contact information is available at: https://pubs.acs.org/10.1021/acs.jpcc.1c01845

\section{Notes}

The authors declare the following competing financial interest(s): The results of this manuscript are also included in a patent application (pending): Application number PCT/ FI2020/050810.

\section{ACKNOWLEDGMENTS}

This work was financially supported by the Academy of Finland (grant numbers 24302332 and 311934). The authors acknowledge the Centre for Material Analysis of the University of Oulu for the use of their facilities and for the fabrication of the electrodes and the CSC - IT Center for Science, Finland, for computational resources. The authors also acknowledge Dr. Marina Tjunina, University of Oulu, for advices on conductivity in ferroelectric oxide perovskites.

\section{REFERENCES}

(1) Damjanovic, D. Ferroelectric, Dielectric and Piezoelectric Properties of Ferroelectric Thin Films and Ceramics. Rep. Prog. Phys. 1998, 61, 1267-1324.

(2) Scott, J. F. Applications of Modern Ferroelectrics. Science 2007, $315,954-959$.

(3) Grinberg, I.; West, D. V.; Torres, M.; Gou, G.; Stein, D. M.; Wu, L.; Chen, G.; Gallo, E. M.; Akbashev, A. R.; Davies, P. K.; Spanier, J. E.; Rappe, A. M. Perovskite Oxides for Visible-Light-Absorbing Ferroelectric and Photovoltaic Materials. Nature 2013, 503, 509-512.
(4) Spanier, J. E.; Fridkin, V. M.; Rappe, A. M.; Akbashev, A. R.; Polemi, A.; Qi, Y.; Gu, Z.; Young, S. M.; Hawley, C. J.; Imbrenda, D.; Xiao, G.; Bennett-Jackson, A. L.; Johnson, C. L. Power Conversion Efficiency Exceeding the Shockley-Queisser Limit in a Ferroelectric Insulator. Nat. Photonics 2016, 10, 611-616.

(5) Paillard, C.; Bai, X.; Infante, I. C.; Guennou, M.; Geneste, G.; Alexe, M.; Kreisel, J.; Dkhil, B. Photovoltaics with Ferroelectrics: Current Status and Beyond. Adv. Mater. 2016, 28, 5153-5168.

(6) Lopez-Varo, P.; Bertoluzzi, L.; Bisquert, J.; Alexe, M.; Coll, M.; Huang, J.; Jimenez-Tejada, J. A.; Kirchartz, T.; Nechache, R.; Rosei, F.; Yuan, Y. Physical Aspects of Ferroelectric Semiconductors for Photovoltaic Solar Energy Conversion. Phys. Rep. 2016, 1-40.

(7) Shafir, O.; Bai, Y.; Juuti, J.; Grinberg, I. Visible-Light-Absorbing Potassium Niobate-Titanate-Molybdate Ferroelectrics. Phys. Rev. Appl. 2020, 14, 44052.

(8) Bai, Y.; Tofel, P.; Palosaari, J.; Jantunen, H.; Juuti, J. A Game Changer: A Multifunctional Perovskite Exhibiting Giant Ferroelectricity and Narrow Bandgap with Potential Application in a Truly Monolithic Multienergy Harvester or Sensor. Adv. Mater. 2017, 29, 1700767.

(9) Balanov, V. A.; Zhao, Z.; Pan, M.; Feldhoff, A.; Bai, Y. Sol-Gel Synthesis and Structural Characterization of Band Gap Engineered Ferroelectric Perovskite Oxide Potassium Sodium Barium Nickel Niobate. J. Sol-Gel Sci. Technol. 2020, 96, 649-658.

(10) Bai, Y.; Xiang, H.; Jantunen, H.; Juuti, J. Multi-Functional Perovskites - an Investigation of Compositional and Processing Influence on Microstructure, Dielectric and Ferroelectric Properties. Eur. Phys. J. Spec. Top. 2019, 228, 1555-1573.

(11) Lou, Q.; Zeng, J.; Man, Z.; Zheng, L.; Park, C.; Kassiba, A.; Liu, Y.; Chen, X.; Li, G. The Effect of Grain Boundary on the Visible Light Absorption of $\mathrm{BaTi}_{1-\mathrm{x}}\left[\mathrm{Ni}_{1 / 2} \mathrm{Nb}_{1 / 2}\right]_{\mathrm{x}} \mathrm{O}_{3-\delta}$ Ferroelectric Ceramics. J. Am. Ceram. Soc. 2019, 102, 7405-7413.

(12) Xiao, H.; Dong, W.; Guo, Y.; Wang, Y.; Zhong, H.; Li, Q.; Yang, M.-M. Design for Highly Piezoelectric and Visible/NearInfrared Photoresponsive Perovskite Oxides. Adv. Mater. 2019, 31, 1805802.

(13) Zhong, H.; Xiao, H.; Jiao, N.; Guo, Y. Boosting Piezoelectric Response of KNN-Based Ceramics with Strong Visible-Light Absorption. J. Am. Ceram. Soc. 2019, 102, 6422-6426.

(14) Bai, Y.; Siponkoski, T.; Peräntie, J.; Jantunen, H.; Juuti, J. Ferroelectric, Pyroelectric, and Piezoelectric Properties of a Photovoltaic Perovskite Oxide. Appl. Phys. Lett. 2017, 110, No. 063903.

(15) Tyunina, M. Conductivity in Ferroelectric Barium Titanate: Electrons versus Oxygen Vacancies. IEEE Trans. Ultrason. Ferroelectr. Freq. Control 2020, 68, 296.

(16) Tyunina, M. Oxygen Vacancies in Perovskite Oxide Piezoelectrics. Materials 2020, 13, 5596.

(17) Rojac, T.; Bencan, A.; Drazic, G.; Sakamoto, N.; Ursic, H.; Jancar, B.; Tavcar, G.; Makarovic, M.; Walker, J.; Malic, B.; Damjanovic, D. Domain-Wall Conduction in Ferroelectric BiFeO3 Controlled by Accumulation of Charged Defects. Nat. Mater. 2017, $16,322-327$.

(18) Malič, B.; Koruza, J.; Hreščak, J.; Bernard, J.; Wang, K.; Fisher, J.; Benčan, A. Sintering of Lead-Free Piezoelectric Sodium Potassium Niobate Ceramics. Materials 2015, 8, 8117-8146.

(19) Song, N.; Momida, H.; Oguchi, T.; Kim, B. G. Polar Phase Transitions and Physical Properties in Fresnoite A2TiSi2O8 (A= Ba, Sr) by First Principles Calculations. J. Solid State Chem. 2016, 242, 136-142.

(20) Kang, Y.; Han, S. Antiperovskite Oxides as Promising Candidates for High-Performance Ferroelectric Photovoltaics: FirstPrinciples Investigation on $\mathrm{Ba} 4 \mathrm{As} 2 \mathrm{O}$ and $\mathrm{Ba} 4 \mathrm{Sb} 2 \mathrm{O}$. ACS Appl. Mater. Interfaces 2020, 12, 43798-43804.

(21) Markov, M.; Alaerts, L.; Miranda, H. P. C.; Petretto, G.; Chen, W.; George, J.; Bousquet, E.; Ghosez, P.; Rignanese, G.-M.; Hautier, G. Ferroelectricity and Multiferroicity in Anti-Ruddlesden-Popper Structures. Pre-print 2020. arxiv.org/abs/2011.09827 
(22) Zheng, T.; Wu, J.; Xiao, D.; Zhu, J. Recent Development in Lead-Free Perovskite Piezoelectric Bulk Materials. Prog. Mater. Sci. 2018, 98, 552-624.

(23) Matsuo, H.; Noguchi, Y.; Miyayama, M. Gap-State Engineering of Visible-Light-Active Ferroelectrics for Photovoltaic Applications. Nat. Commun. 2017, 8, 207. 